\title{
Case Report \\ Achromobacter xylosoxidans subsp. xylosoxidans prosthetic aortic valve infective endocarditis and aortic root abscesses
}

Correspondence

S. van $\mathrm{Hal}$

vanhal@gotalk.net.au

Received 5 July 2007

Accepted 3 December 2007

\author{
S. van Hal, D. Stark, D. Marriott and J. Harkness \\ Department of Microbiology, St Vincent's Hospital, Darlinghurst 2010, NSW Sydney, Australia
}

We report a case of prosthetic valve infective endocarditis and aortic root abscesses caused by Achromobacter xylosoxidans subsp. xylosoxidans. The patient was an intravenous drug user and had injected amphetamines using 'duck pond water' as a diluent. After surgical intervention and 6 weeks of intravenous meropenem therapy, the patient made an uneventful recovery.

\section{Case report}

A 37-year-old male was transferred from a peripheral hospital to St Vincent's Hospital, Sydney, Australia, for surgical management of an aortic root abscess and prosthetic valve endocarditis (PVE). The patient's native aortic valve (AV) was replaced in November 2006 secondary to a meticillin-sensitive Staphylococcus aureus $\mathrm{AV}$ endocarditis following episodes of intravenous drug use. Following AV replacement, the patient returned to his normal activities. However, he denied any further intravenous drug use. A follow-up transthoracic echocardiogram in March 2007 revealed a normally functioning AV. Two months later (May 2007), the patient presented to a rural hospital with fevers, hypotension and congestive cardiac failure. A diagnosis of PVE was considered and intravenous gentamicin, ceftriaxone and vancomycin were commenced. However, the symptoms of sepsis and congestive cardiac failure failed to improve with medical therapy and the patient was transferred for surgical intervention. A repeat AV replacement and a homograft aortic root replacement were performed for PVE and multiple aortic root abscesses.

Blood cultures and tissue from the $\mathrm{AV}$ and root were sent for microbiological analysis. An oxidase-positive Gramnegative rod was isolated from both tissue and blood culture after $48 \mathrm{~h}$ of incubation. The Gram-negative rod from the blood culture was identified as Burkholderia cepacia by an API 20 NE (bioMérieux) with $99.5 \%$ probability and a 'very good identification' confidence level (biotype profile number 1047777), while the Gramnegative rod from the tissue cultures was identified as Achromobacter xylosoxidans by an API 20 NE (bioMérieux) with $94.5 \%$ probability (biotype profile number 1040477) and a 'good identification' confidence level. Identical phenotypic results were obtained on repeat API $20 \mathrm{NE}$

Abbreviation: $\mathrm{AV}$, aortic valve. testing on fresh subcultures from both the blood culture and the tissue isolates.

Due to identification discrepancies, further testing by analysis of whole-cell long-chain fatty acids and 16S rRNA were performed on both isolates. The Microbial Identification System (MIDI Corporation) confirmed both isolates to be A. xylosoxidans subsp. xylosoxidans (similarity index of 0.658 ; an index $>0.5$ is considered adequate for speciation). To exclude the possibility of the B. cepacia complex, a PCR was performed as previously described using primers targeting the $r e c A$ gene (Mahenthiralingam et al., 2000). The PCR was negative for B. cepacia complex DNA.

Genomic DNA was extracted from both isolates using a QIAamp DNA Mini Kit (QIAGEN), and DNA amplification of the small-subunit rRNA gene was performed using universal primers as previously described by Fry et al. (2005). The PCR products were purified using the QIAquick PCR Purification kit (Qiagen) according to the manufacturer's instructions. The PCR products were sequenced in both directions on an ABI Prism 3730 automated sequencer at the SUPAMAC facility (Royal Prince Alfred Hospital, Sydney). The sequences generated were compared to those available in the GenBank databases using the BLASTN program run on the National Center for Biotechnology Information server (http://www.ncbi.nlm. nih.gov/BLAST/).

The sequence data generated from the 16S rRNA gene from both isolates were identical and demonstrated $100 \%$ similarity with the 16S rRNA gene sequence of numerous A. xylosoxidans subsp. xylosoxidans isolates (GenBank accession nos DQ466568, DQ361075, AF411021, AF411020, AF411019 and AB161691). The organism revealed the following susceptibilities by E-test: gentamicin MIC $0.094 \mu \mathrm{g} \mathrm{ml}{ }^{-1}$; ticarcillin-clavulanate MIC $12 \mu \mathrm{g}$ $\mathrm{ml}^{-1}$; cefepime MIC $0.016 \mu \mathrm{g} \mathrm{ml}^{-1}$; cefotaxime MIC $12 \mu \mathrm{g}$ $\mathrm{ml}^{-1}$, ciprofloxacin MIC $0.75 \mu \mathrm{g} \mathrm{ml}^{-1}$; and meropenem 
MIC $0.38 \mu \mathrm{g} \mathrm{ml}^{-1}$. The patient was treated with meropenem for 6 weeks and made an uneventful recovery.

On further questioning, the patient admitted to injecting intravenous drugs 1 week prior to his presentation. Furthermore, the patient described a febrile illness immediately after injecting amphetamines which spontaneously resolved several hours later. The probable source of infection was established when the patient described the process of injecting. He was undertaking a long drive and decided to inject amphetamines to keep awake. He stopped at a friend's house and obtained water from his duck pond, which he used to solubilize the drug for injection without filtering or boiling.

\section{Discussion}

The family Alcaligenaceae comprises several genera, based on phenotypic and genotypic data, including the genus Achromobacter (Yabuuchi et al., 1998). A. xylosoxidans is a ubiquitous environmental Gram-negative, oxidase-positive, non-glucose-fermenting rod. There are two subspecies, A. xylosoxidans subsp. xylosoxidans and A. xylosoxidans subsp. denitrificans.

Human infections are rare. Nosocomial infections predominate with an association between infection and immunosuppression, especially in patients with underlying malignancy, in HIV-infected patients and in premature infants (Aisenberg et al., 2004; Manfredi et al., 1997). Contaminated intravenous lines are the most common source of infection, with other potential sources including dialysis fluid, incubators and mechanical ventilators (Gómez-Cerezo et al., 2003; Reverdy et al., 1984). Community-acquired infections account for the minority of cases and are generally restricted to cystic fibrosis patients with temporary or persistent infections of the respiratory tract (Davies \& Rubin, 2007).

The most common manifestation of infection with this organism is bacteraemia. Polymicrobial bacteraemia occurs in $28 \%$ of patients, with coagulase-negative Staphylococcus the most common accompanying organism (Gómez-Cerezo et al., 2003). Endocarditis has been described on four previous occasions only (Ahn et al., 2004; Duggan et al., 1996; Lofgren et al., 1981; Martino et al., 1990). Our patient is unique as infective endocarditis was secondary to a community-acquired bacteraemia as a consequence of intravenous injection of drugs. The previously reported cases required removal of the pacemaker leads and prosthetic valve. The virulence of this organism was confirmed in our patient, who required an $\mathrm{AV}$ and root replacement. The importance of an accurate medical history to ascertain the source of unusual organisms is highlighted.

Characteristic antimicrobial susceptibility patterns of $A$. xylosoxidans subsp. xylosoxidans include high levels of resistance to cephalosporin $(>90 \%)$, aminoglycoside $(>90 \%)$ and quinolone $(>80 \%)$ antibiotics. Variable intrinsic resistance to broad-spectrum anti-pseudomonal penicillins is described. Although our isolate was sensitive to ticarcillin-clavulanic acid on MIC testing, a $\beta$-lactamase enzyme was detected by nitrocephin disc testing; therefore, meropenem was chosen as resistance to this antibiotic is rare (Gómez-Cerezo et al., 2003; Shie et al., 2005).

Identification of A. xylosoxidans subsp. xylosoxidans may be problematic. Traditional phenotypic and commercially available tests are often unreliable and may lead to misidentification as other non-fermenting organisms, in particular members of the B. cepacia complex (Wellinghausen et al., 2006). Tissue and blood isolates which were subsequently proven to be identical gave different profiles and hence a different identification using the API $20 \mathrm{NE}$ system. Molecular methods such as sequencing of ribosomal genes allow for more accurate identification of non-fermenting Gram-negative rods than traditional phenotypic methods. However, care should be taken when interpreting the sequence data generated as public DNA databases such as GenBank may have faulty or incorrectly assigned sequences leading to the misidentification of isolates (Wellinghausen et al., 2006).

A. xylosoxidans bacteraemia has a mortality rate of between 15 and $48 \%$ (Duggan et al., 1996; Shie et al., 2005). Risk factors for higher mortality rates include age over 65 years, neutropenia, presence of polymicrobial infection and nosocomial infection (Gómez-Cerezo et al., 2003; Shie et al., 2005).

In conclusion, community-acquired A. xylosoxidans bacteraemia is uncommon while endocarditis is rare. An accurate intravenous drug use history should be sought, especially related to the paraphernalia and methods used in the injecting process. The most appropriate antimicrobial therapy has not been determined but our patient responded well to surgical intervention and meropenem.

\section{Acknowledgements}

We thank Marion Yeun and Maureen Lynch from the Identification Reference Laboratory, Centre for Infectious Diseases and Microbiology, Institute of Clinical Pathology and Medical Research, Westmead Hospital, NSW, Australia, for help with analysis of wholecell long-chain fatty acid testing of the isolate.

\section{References}

Ahn, Y., Kim, N. H., Shin, D. H., Park, O. Y., Kim, W., Jeong, M. H., Cho, J. G., Park, J. C. \& Kang, J. C. (2004). Pacemaker lead endocarditis caused by Achromobacter xylosoxidans. J Korean Med Sci 19, 291-293.

Aisenberg, G., Rolston, K. V. \& Safdar, A. (2004). Bacteremia caused by Achromobacter and Alcaligenes species in 46 patients with cancer (1989-2003). Cancer 101, 2134-2140.

Davies, J. C. \& Rubin, B. K. (2007). Emerging and unusual gramnegative infections in cystic fibrosis. Semin Respir Crit Care Med 28, 312-321.

Duggan, J. M., Goldstein, S. J., Chenoweth, C. E., Kauffman, C. A. \& Bradley, S. F. (1996). Achromobacter xylosoxidans bacteremia: report of four cases and review of the literature. Clin Infect Dis 23, 569-576. 
Fry, N. K., Duncan, J., Malnick, H., Warner, M., Smith, A. J., Jackson, M. S. \& Ayoub, A. (2005). Bordetella petrii clinical isolate. Emerg Infect Dis 11, 1131-1133.

Gómez-Cerezo, J., Suárez, I., Ríos, J. J., Peña, P., García de Miguel, M. J., de José, M., Monteagudo, O., Linares, P., Barbado-Cano, A. \& Vázquez, J. J. (2003). Achromobacter xylosoxidans bacteremia: a 10-year analysis of 54 cases. Eur J Clin Microbiol Infect Dis 22, 360-363.

Lofgren, R. P., Nelson, A. E. \& Crossley, K. B. (1981). Prosthetic valve endocarditis due to Achromobacter xylosoxidans. Am Heart J 101, 502.

Mahenthiralingam, E., Bischof, J., Byrne, S. K., Radomski, C., Davies, J. E., Av-Gay, Y. \& Vandamme, P. (2000). DNA-based diagnostic approaches for identification of Burkholderia cepacia complex, Burkholderia vietnamiensis, Burkholderia multivorans, Burkholderia stabilis, and Burkholderia cepacia genomovars I and III. J Clin Microbiol 38, 3165-3173.

Manfredi, R., Nanetti, A., Ferri, M. \& Chiodo, F. (1997). Bacteremia and respiratory involvement by Alcaligenes xylosoxidans in patients infected with the human immunodeficiency virus. Eur J Clin Microbiol Infect Dis 16, 933-938.
Martino, P., Micozzi, A., Venditti, M., Gentile, G., Girmenia, C., Raccah, R., Santilli, S., Alessandri, N. \& Mandelli, F. (1990). Catheterrelated right-sided endocarditis in bone marrow transplant recipients. Rev Infect Dis 12, 250-257.

Reverdy, M. E., Freney, J., Fleurette, J., Coulet, M., Surgot, M., Marmet, D. \& Ploton, C. (1984). Nosocomial colonization and infection by Achromobacter xylosoxidans. J Clin Microbiol 19, 140-143.

Shie, S. S., Huang, C. T. \& Leu, H. S. (2005). Characteristics of Achromobacter xylosoxidans bacteremia in northern Taiwan. J Microbiol Immunol Infect 38, 277-282.

Wellinghausen, N., Wirths, B. \& Poppert, S. (2006). Fluorescence in situ hybridization for rapid identification of Achromobacter xylosoxidans and Alcaligenes faecalis recovered from cystic fibrosis patients. J Clin Microbiol 44, 3415-3417.

Yabuuchi, E., Kawamura, Y., Kosako, Y. \& Ezaki, T. (1998). Emendation of genus Achromobacter and Achromobacter xylosoxidans (Yabuuchi and Yano) and proposal of Achromobacter ruhlandii (Packer and Vishniac) comb. nov., Achromobacter piechaudii (Kiredjian et al.) comb. nov., and Achromobacter xylosoxidans subsp. denitrificans (Ruger and Tan) comb. nov. Microbiol Immunol 42, 429-438. 